BPC 01171

\title{
Fractional exponential decay of a membrane protein population due to capture by coated pits
}

\author{
F.W. Wiegel and J.H.J. van Opheusden \\ Center for Theoretical Physics, Twente University of Technology, P.O. Box 217, Enschede 7500 AE, The Netherlands
}

Received 28 July 1986

Revised manuscript received 19 April 1987

Accepted 23 April 1987

Membrane protein; Coated pit; Receptor diffusion; Fractional exponential decay

\begin{abstract}
We consider the lateral diffusion of receptors, or other membrane proteins, in the outer membranes of certain cells, and their capture by coated pits. It is shown, for the case in which the coated pits are in fixed random positions, that the long-time decay of the total number of uncaptured proteins is of the fractional exponential form, $N(t)=N_{0} \exp \{-\sqrt{(t / \tau)}\}$, and not of the pure exponential form, $N(t)=N_{0} \exp (-t / \tau)$, which is usually assumed.
\end{abstract}

\section{Introduction}

In a variety of biophysical experiments one observes the decay of a population of membrane proteins due to their capture by some system of traps. The membrane proteins diffuse laterally in the cell membrane, and the traps are in random, but fixed positions in the membrane. An important example is the lateral diffusion of receptors in the membrane of basophils or mast cells, and their capture by coated pits.

These experiments are usually interpreted under the assumption that the total number $N(t)$ of uncaptured proteins at time $t$ will decay with a pure exponential

$N(t)=N_{0} \exp (-t / \tau)$,

provided we look at the long-time behavior of the capture process. Here, $\tau$ is a constant with the dimension of time, which is essentially equal to the mean lifetime of a membrane protein.

Correspondence address: F.W. Wiegel, Center for Theoretical Physics, Twente University of Technology, P.O. Box 217, Enschede 7500 AE, The Netherlands.
In this paper we analyze a simple model in which the traps are in fixed but completely random positions. We shall show that the long-time decay of the protein population is not of the pure exponential form (eq. 1), but rather of the fractional exponential form

$N(t)=N_{0} \exp \left\{-(t / \tau)^{1 / 2}\right\}$

Before turning to the details of the calculation in section 2, we should point out that fractional exponential relaxation of the form given by eq. 2 , but with various powers between 0 and 1 , is found experimentally in a great variety of complex systems. These include mechanical relaxation, dielectric relaxation, relaxation of ionic conductivity, spin-lattice relaxation, luminescence relaxation and various relaxation processes in electronic hopping, magnetic systems and in systems consisting of entangled polymers. For an overview of both the experimental and theoretical work in this field the reader is referred to the proceedings of a recent conference [1]. At the time of writing there is no consensus as to why the fractional exponential form of relaxation is so universal that it ap- 
pears in physical systems of the most diverse nature, although one of us has speculated that it simply reflects a universal property of the heat bath [2]. The present two-dimensional model for membrane protein capture by randomly distributed coated pits has the advantage that the physical origin of this 'anomalous' behavior can be shown quite clearly and that the theory can be developed analytically in considerable detail.

\section{Some physical considerations}

Consider a cell membrane of area $A$ in which $M$ coated pits or traps of some type are placed in fixed but random positions, i.e., each coated pit can be located anywhere on $A$ with a surface probability density $1 / A$. Each coated pit is modeled as a perfectly absorbing circular region of radius $a$. At $t=0$ one distributes $N_{0}$ receptors (or membrane proteins of some type) uniformly throughout $A$; they diffuse laterally in the membrane and become absorbed instantaneously when they touch any coated pit. The desired quantity is then an expression for the long-time decay of the number $N(t)$ of free proteins at time $t$.

In order to grasp the basic physics of this relaxation process, one should note an essential difference between these coated pits and those that are placed in regular positions: The former tend to cluster in patches in which their number density is somewhat higher than the average

$m=\frac{M}{A}$

moreover, in between these patches one finds isolated regions ('holes') in which there are no coated pits at all (fig. 1). So, one can visualize the system of random coated pits as a more or less uniform background with density (eq. 3), in which one finds, in various locations, holes of various shape and size.

The probability of finding a hole of area $\alpha$ can be calculated if one notes that the positions of the coated pits are statistically independent. Hence, the probability that the first coated pit is located outside the area $\alpha$ equals $(1-\alpha / A)$, and the probability that this holds for all traps is simply the

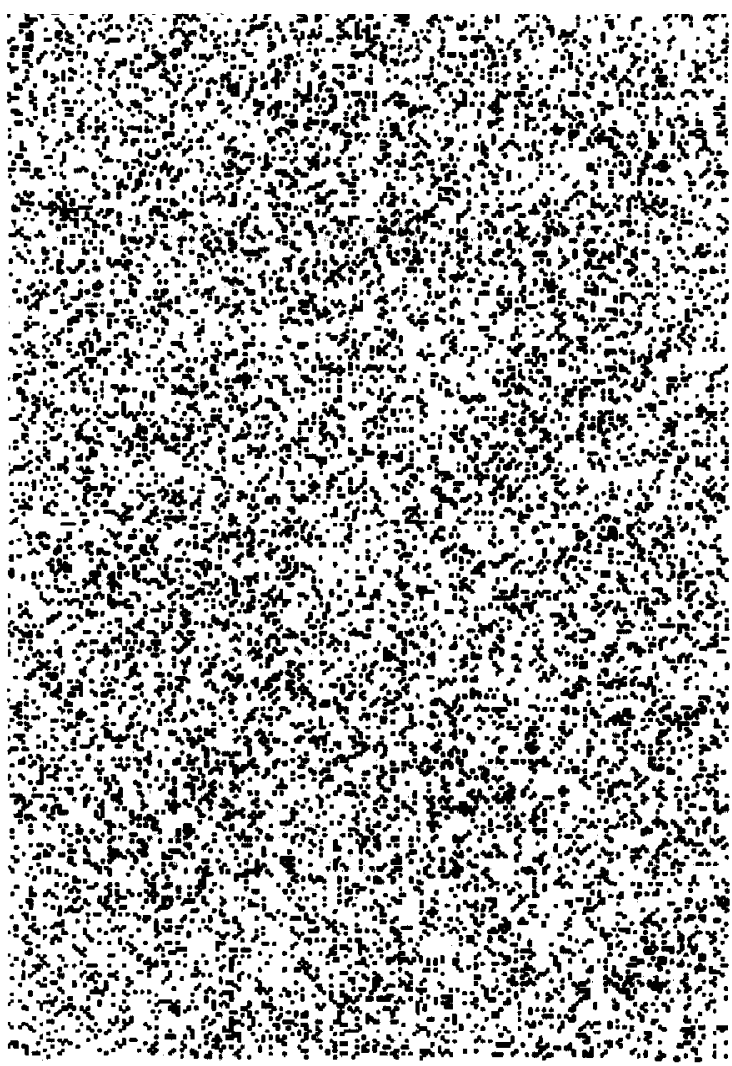

Fig. 1. Random distribution of coated pits in a planar membrane. Each coated pit is represented by a small dot. One can observe regions in which a relatively dense clustering of dots appears, as well as rather large holes, in which there are no coated pits at all. It is mainly the diffusion of receptor proteins from these holes that gives rise to the fractional exponential decay law for the total number of receptors.

$\boldsymbol{M}$-th power of this expression. Furthermore, we shall assume that the shape of the larger holes is circular, with $\alpha=\pi s^{2}$, so the probability that we find a circular area devoid of traps with at least one of the $M$ traps in a neighbourhood ds of the circumference, and the other $M-1$ traps outside is

$$
\begin{aligned}
(\text { probability })= & \text { constant } \times M(2 \pi s \mathrm{~d} s / A) \\
& \times\left(1-\pi s^{2} / A\right)^{M-1}
\end{aligned}
$$

After taking the thermodynamic limit ( $M \rightarrow$ $\infty, A \rightarrow \infty, m=M / A$ constant), the total number $H(s) \mathrm{d} s$ of holes with radius between $s$ and 
$s+\mathrm{d} s$ becomes

$H(s)=2 \pi m s H_{0} \exp \left(-\pi m s^{2}\right)$.

Hence $H_{0}$, which equals the total number of holes in the system, will be proportional to the total area of the system. It is the only adjustable parameter in the present theory.

\section{Decay of protein population from a single hole}

Now consider a circular hole of radius $s$, surrounded by a more or less uniform distribution of coated pits with a surface density $m$, and follow only those proteins which at $t=0$ are situated inside this hole. At $t=0$ this population has a concentration (number per unit area) which is equal to $c_{0}$ inside the hole and zero outside it. In this section we calculate the decay of this population asymptotically for $t \rightarrow \infty$.

Let the protein concentration for $t>0$ be denoted by $c(r, t)$ where one uses polar coordinates around the center of the hole. If the lateral diffusion of the proteins in the membrane is described by a single constant $D$, this function is the solution of the equations

$$
\begin{array}{ll}
\frac{\partial c}{\partial t}=D \Delta c, & (0<r<s), \\
\frac{\partial c}{\partial t}=D \Delta c-2 \pi D m c, & (r>s) .
\end{array}
$$

Here $\Delta=\mathrm{d}^{2} / \mathrm{d} r^{2}+(1 / r) \cdot \mathrm{d} / \mathrm{d} r$ is the Laplace operator in two dimensions and an extra logarithmic dependence of the term $2 \pi D m c$ on $m$ and $a$ has been neglected (cf. the discussion in ref. 3 ).

The general solution of eq. 6 has the form of an eigenfunction expansion. If the ground state $\phi_{0}(r)$, with eigenvalue $\lambda_{0}$, is a bound state, the long-time behavior of the solution is

$c(r, t)=d_{0} \phi_{0}(r) \exp \left(-\lambda_{0} t\right),(t \rightarrow \infty)$,

where $d_{0}$ is a constant. The protein population $N(s, t)$ will decay according to

$N(s, t)=\pi s^{2} c_{0} \exp \left(-\lambda_{0} t\right)$,

for long times. We now proceed to calculate the lowest eigenvalue $\lambda_{0}(s)$, which sets the scale for this relaxation process.
The ground state should be solved from

$$
\begin{aligned}
& D\left(\frac{\mathrm{d}^{2}}{\mathrm{~d} r^{2}}+\frac{1}{r} \frac{\mathrm{d}}{\mathrm{d} r}\right) \\
& \quad \times \phi_{0}+\lambda_{0} \phi_{0}=0, \quad(0<r<s), \\
& D\left(\frac{\mathrm{d}^{2}}{\mathrm{~d} r^{2}}+\frac{1}{r} \frac{\mathrm{d}}{\mathrm{d} r}\right) \\
& \quad \times \phi_{0}-\left(2 \pi D m-\lambda_{0}\right) \phi_{0}=0, \quad(r>s) .
\end{aligned}
$$

There are boundary conditions at $r=0, r=s$ and $r=\infty$. The boundary condition at $r=0$ requires $\phi_{0}$ to be finite, hence

$\phi_{0}(r)=B_{1} J_{0}\left(r \sqrt{\frac{\lambda_{0}}{D}}\right),(0<r<s)$

where $J_{0}$ is the Bessel function of the first kind (cf. chapter 9 of ref. 4). The boundary condition at $r=\infty$ requires $\phi_{0}$ to vanish, hence

$\phi_{0}(r)=B_{2} K_{0}\left(r \sqrt{2 \pi m-\frac{\lambda_{0}}{D}}\right),(r>s)$,

where $K_{0}$ is the modified Bessel function. The values of the two constants $B_{1}$ and $B_{2}$ follow from the boundary conditions at $r=s$ where $\phi_{0}$ and $\mathrm{d} \phi_{0} / \mathrm{d} r$ must be continuous. This gives the set of conditions

$$
\begin{gathered}
B_{1} J_{0}\left(s \sqrt{\frac{\lambda_{0}}{D}}\right)=B_{2} K_{0}\left(s \sqrt{2 \pi m-\frac{\lambda_{0}}{D}}\right) \\
B_{1} J_{1}\left(s \sqrt{\frac{\lambda_{0}}{D}}\right) \sqrt{\frac{\lambda_{0}}{D}} \\
=B_{2} K_{1}\left(s \sqrt{2 \pi m-\frac{\lambda_{0}}{D}}\right) \sqrt{2 \pi m-\frac{\lambda_{0}}{D}}
\end{gathered}
$$

There are various ways to proceed from here. First, one should note that the membrane proteins in small holes are captured very rapidly. Hence, the longtime behavior of the relaxation function is determined by the decay rate from the large holes. For these large holes $\lambda_{0} / D$ will be of order $s^{-2}$ and, hence, small as compared to $2 \pi \mathrm{m}$. In this case one can replace the quantity $\sqrt{2 \pi m-\lambda_{0} / D}$ by $\sqrt{2 \pi m}$ on the right-hand sides of eqs. 11a and 
11b. Taking the ratio one finds

$\xi \frac{J_{1}(\xi)}{J_{0}(\xi)} \cong s \sqrt{2 \pi m} \frac{K_{1}(s \sqrt{2 \pi m})}{K_{0}(s \sqrt{2 \pi m})}$,

where

$\xi=s \sqrt{\frac{\lambda_{0}}{D}}$

The left-hand side of eq. 12 increases monotonically from 0 to $\infty$ for $0<\xi<j_{0,1}$, where $j_{0,1}=$ 2.4048 is the first zero of $J_{0}(\xi)$. Hence, for

$s \sqrt{2 \pi m} \gg 1$

the solution of eq. 12 will be very close to $\xi=j_{0,1}$, or

$\lambda_{0}=j_{0,1}^{2} \frac{D}{s^{2}}$.

Combination of eqs. 8 and 15 gives the asymptotic form of the decay law for the population from one large hole of radius $s$

$N(s, t)=\pi s^{2} c_{0} \exp \left(-j_{0.1}^{2} \frac{D t}{s^{2}}\right)$,

which will be used in section 4 to determine the decay law of the total membrane protein population.

\section{Fractional exponential decay of the total popula- tion}

The total population of uncaptured membrane proteins at time $t$ is given by the expression

$N(t)=\int_{0}^{\infty} N(s, t) H(s) \mathrm{d} s$.

Substitution of eqs. 5 and 16 gives, for the longtime behavior,

$$
\begin{aligned}
N(t)= & 2 \pi^{2} \dot{c_{0}} m H_{0} \int_{s_{0}}^{\infty} s^{3} \\
& \times \exp \left(-\pi m s^{2}-j_{0,1}^{2} \frac{D t}{s^{2}}\right) \mathrm{d} s,
\end{aligned}
$$

where $s_{0}$ denotes a lower cut-off on the size of holes, which is of order $m^{-1 / 2}$. It is straightfor- ward to use the saddlepoint method to show that for long times the main contribution to the integral comes from the vicinity of the maximizing $s$, which grows with time proportional to $t^{1 / 4}$. Hence, the lower integration limit in eq. 18 may be put at $s_{0}=0$. In this case the integral happens to be given exactly by

$N(t)=2 \pi c_{0} H_{0} j_{0,1}^{2} D t K_{2}\left(2 j_{0,1} \sqrt{\pi m D t}\right)$,

where we used eq. 3.471 .9 of ref. 5. Using the asymptotic formula for the modified Bessel function $K_{2}$ for $t \rightarrow \infty$, one finds [4]

$$
\begin{aligned}
N(t)= & \pi^{5 / 4} c_{0} H_{0} j_{0,1}^{3 / 2} m^{-1 / 4}(D t)^{3 / 4} \\
& \times \exp \left(-2 j_{0,1} \sqrt{\pi m D t}\right)
\end{aligned}
$$

Of course, the prefactor $t^{3 / 4}$ is meaningless as compared to the exponential factor.

\section{Concluding remarks}

We have demonstrated a fractional exponential decay of the total membrane protein population, due to capture by coated pits (eqs. 19 and 20). From the derivation of this result it should be clear that one expects the fractional exponential relaxation to appear in all cases in which the positions of the coated pits are sufficiently random to allow the occurrence of large empty regions.

Ideas which differ from those in the present paper have been used by Ovchinnikov and Zeldovich [6] to study bimolecular reaction kinetics in three dimensions. The two-dimensional system studied here obeys a different decay law than the analogous three-dimensional system of random traps [7].

It should be noted here that Torney (personal communication) has performed a numerical simulation of the two-dimensional system which forms the subject of this paper. His results seem to confirm a decay law of the form given by eq. 19 .

We would finally like to point out that eq. 19 has the form of a fractional exponential of the type studied by various authors (a review of the theoretical work can be found in ref. 8). For the model studied here the physical origin of this 
decay law was quite easy to understand; whether equally simple mechanisms are relevant in other cases remains to be shown.

\section{Acknowledgements}

The work of J.H.J. v. O. is part of the research program of the Stichting Fundamenteel Onderzoek der Materie (FOM - 82.82.255), which is financially supported by the Nederlandse Organisatie voor Zuiver-Wetenschappelijk Onderzoek (ZWO). F.W.W. has profited from discussions with Alan S. Perelson, which were made possible by NATO Collaborative Research Grant 86/719.

\section{References}

1 K.L. Ngai and G.B. Wright, Relaxations in complex systems (Office of Naval Research, Arlington, VA, 1985).

2 A.K. Rajagopal and F.W. Wiegel, Physica 127A (1984) 218.

3 F.W. Wiegel and C. DeLisi, Am. J. Physiol. 12 (1982) R475.

4 M. Abramowitz and I.A. Stegun, Handbook of mathematical functions (Dover, New York, 1970).

5 I.S. Gradshteijn and P.M. Ryzhik, Table of integrals, series and products (Academic Press, New York, 1965).

6 A.A. Ovchinnikov and Y.B. Zeldovich, Chem. Phys. 28 (1978) 215.

7 F.W. Wiegel, Physica 139A (1986) 209.

8 A.K. Rajagopal and K.L. Ngai, in: Relaxations in complex systems, eds. K.L. Nagai and G.B. Wright (Office of Naval Research, Arlington, VA, 1985) p. 275. 